\title{
Écriture et échec dans l'œuvre de Diamela Eltit
}

\section{Catherine Pélage}

\section{OpenEdition}

Journals

Édition électronique

URL : https://journals.openedition.org/cher/11989

DOI : $10.4000 /$ cher.11989

ISSN : 2803-5992

\section{Éditeur}

Presses universitaires de Strasbourg

\section{Édition imprimée}

Date de publication : 30 juin 2012

Pagination : 27-38

ISBN : 978-2-35410-046-9

ISSN : 1968-035X

\section{Référence électronique}

Catherine Pélage, «Écriture et échec dans l'œuvre de Diamela Eltit », reCHERches [En ligne], 8| 2012, mis en ligne le 21 février 2022, consulté le 24 février 2022. URL : http://journals.openedition.org/cher/ 11989 ; DOI : https://doi.org/10.4000/cher.11989

\section{cc) (i)}

Ce(tte) œuvre est mise à disposition selon les termes de la Licence Creative Commons Attribution Pas d'Utilisation Commerciale - Partage dans les Mêmes Conditions 4.0 International. 


\title{
Écriture et échec dans l'œuvre de Diamela Eltit
}

\author{
Catherine Pélage \\ Université d'Orléans
}

\begin{abstract}
iamela Eltit a fait son apparition sur la scène publique chilienne durant la dictature d'Augusto Pinochet. Sa démarche s'inscrivait dans les actions menées par le Groupe CADA, Colectivo de Acciones De Arte, qui réunissait, depuis 1979, différents artistes; ceux-ci, par le biais de ce qu'ils appelaient des «actions d'art» remettaient en cause l'ordre dictatorial. Lors de ses "performances", Diamela Eltit se mettait en scène dans des maisons closes ou des quartiers défavorisés, questionnant, depuis les marges, les discours et la morale officiels. Elle a publié ses premières œuvres sous la dictature: la violence dictatoriale et la destruction tant individuelle que collective qu'elle a générée est présente dans des romans comme Lumpérica de 1983 ou El cuarto mundo de 1988. Or, malgré l'évolution politique du Chili, l'échec des personnages et de la société reste omniprésent, que ce soit dans El Padre Mío publié en 1989 durant la transition démocratique ou dans Mano de obra, édité en 2002.

Cette constante de l'échec malgré un retour à la démocratie est liée, selon la conception de Diamela Eltit, aux séquelles que la dictature a laissées dans la société chilienne. A son avis, la finalité du coup d'État militaire ne fut pas idéologique mais économique. Aussi explique-t-elle:

Lo que a veces está un poco soterrado o escondido en el golpe es que fue una alianza entre la derecha económica y los militares. La finalidad del golpe de estado fue restituir los bienes. El golpe es un proyecto económico. Las muertes, la represión, la violencia, el estado de excepción fueron hechos para el capital. En ese sentido, el golpe no es ideológico. El golpe es un proyecto económico (Lazzara 2002: 122).
\end{abstract}


Selon elle, la violence économique mise en place sous le régime d'Augusto Pinochet s'est enracinée dans la société et continue ses ravages par le biais de ce qu'elle appelle "un néo-libéralisme effréné». D'où son affirmation: «habría que establecer un viaje desde el silencio más elocuente que buscó la cultura dictatorial hasta llegar al desaforado ímpetu de la cultura comercial» (Carreño 2009: 346).

Or, il nous semble que l'œuvre de Diamela Eltit peut être interprétée comme un voyage depuis le silence imposé par la culture dictatoriale jusqu'à la domination exclusive du marché. Ainsi, dans son œuvre, l'échec des personnages reflète un échec politique et social tandis que les réflexions des narrateurs soulignent des difficultés d'expression. Nous pourrions arriver à un constat d'échec revendiqué par l'auteure. Mais, il me semble que de cet échec des personnages et des narrateurs naît une forme d'écriture qui place le lecteur en situation d'échec de façon à ouvrir de nouvelles pistes de réflexion. L'étude du roman Mano de obra servira de point d'ancrage dans notre appréhension de la démarche de Diamela Eltit.

\section{Ecriture et échec dans Mano de obra}

Dans le roman Mano de obra, publié en 2002, l'espace dominant est celui d'un supermarché et les narrateurs sont tous des employés de la grande surface. Le premier chapitre, intitulé «El despertar de los trabajadores (Iquique, 1911)», reflète un enfermement croissant du narrateur dans son lieu de travail aliénant. La seconde partie, "Puro Chile (Santiago, 1970)» montre l'exploitation et la marginalisation croissante de la main d'œuvre.

La première partie est le fait d'un narrateur autodiégétique. Ce dernier narre sa vie quotidienne au supermarché, lieu de travail aliénant dans lequel il passe le plus clair de son temps. Cet enfermement spatial engendre une profonde aliénation chez l'employé-narrateur. La présence du champ lexical de l'enfermement dans son discours est flagrante. Celui-ci se sent par exemple «desesperado ante una injusta reclusión». L'exploitation économique dont il est victime provoque une vision dégradée de lui-même visible dans des affirmations du type:

Esto pienso:

«Es posible que no merezca que los clientes me traten tan mal.» Pero no lo pienso enteramente. En realidad no. No enteramente (Eltit 2002: 262). 
D'où une idée d'impuissance renforcée par l'utilisation de certaines références bibliques. C'est le cas de la pomme qui revêt une dimension particulière dans les crises existentielles du personnage. Ce dernier déclare: "No estoy enfermo (en realidad) sino que me encuentro inmerso en un viaje de salida de mí mismo. Ordeno una a una las manzanas. Ordeno una a una las manzanas. Ordeno una a una las manzanas. Ordeno una a una (las manzanas)» (282).

La pomme n'évoque donc pas l'arbre de la connaissance du bien et du mal mais un travail répétitif et obsédant. L'employé, tel un Sisyphe moderne, est condamné à faire et refaire éternellement la pile de pommes que les clients défont. Le protagoniste n'essaie jamais de transgresser les interdits qui lui sont fixés; néanmoins, il se voit progressivement fragilisé, le premier chapitre se terminant sur une allusion à son licenciement, autrement dit à son expulsion d'un lieu qui était loin d'être comparable au paradis terrestre.

Par ailleurs, l'aliénation sociale influe sur le mode d'expression du narrateur. Celui-ci semble doté d'une fonction de communication. Un certain nombre d'onomatopées paraissent être des marques d'oralité. D'autre part, les multiples parenthèses ont à première vue la fonction de clarifier, pour autrui, le contenu des phrases. Cependant, elles peuvent être interprétées différemment. L'isolement de l'employé est évident. Au sein d'un discours que nul n'écoute, les parenthèses ne constituent-elles pas des parties plus inaudibles encore? Les difficultés d'expression du narrateur sont telles que certaines phrases interrogatives sont dépourvues de point d'interrogation, ce qui crée une impression d'atonie, reflet peut-être de questions qui savent qu'elles resteront sans réponse.

Il est bien évident que l'enfermement spatial et pathologique du narrateur autodiégétique est le reflet d'une société malade. Le chapitre "El obrero gráfico (Valparaíso, 1926)» est révélateur de ce phénomène. A l'occasion des fêtes de Noël, le narrateur, déguisé, représente l'un des personnages d'une crèche. Le décalage entre le rôle à jouer et la réalité quotidienne provoque l'hilarité des employés: "Juntos, debemos esconder la carcajada que nos suscita la representación de una familia, (¿qué me dicen, ah? ¿Qué?)» (290).

Cette scène met en relief le monde artificiel créé par le supermarché: l'enfant Jésus et les animaux sont en plastique, les employés sont déguisés, les lumières, artificielles, et les fêtes religieuses, un argument de vente. Par ailleurs, la structure familiale est désormais inexistante mais rien de solide 
ne l'a remplacée. L'être humain est donc d'une vulnérabilité extrême et livré en pâture à la société de consommation:

El año nuevo se retira colmado de divisas. Próspero año y yo aquí, de pie en el súper, cautelando la estricta circulación de la moneda [...]. Inclinado, curvado por las peticiones, me abrazo locamente a los estantes y celebro mi año (nuevo), mi triunfo y mi silencio (195).

De façon fort symbolique, le chapitre se clôt à l'aube d'une nouvelle année puis la seconde partie montre une aggravation de l'exploitation de la main d'œuvre et une marginalisation croissante des narrateurs.

Dans la seconde partie, intitulée «Puro Chile (Santiago 1970)» une voix narrative plurielle prend la parole. Il s'agit d'un "nosotros» aux contours indéfinis, au sein duquel émergent des personnages dont les prénoms apparaissent dès les sous-titres: Gloria, Alberto, Enrique, Gabriel. L'étude de l'enchaînement des premiers sous-chapitres est éclairante: les membres du groupe accompagnent Isabel, fatiguée par son travail, à un entretien avec son superviseur. Ils l'attendent à l'extérieur du bureau tout en sachant que son supérieur hiérarchique est en train d'abuser d'elle. Le harcèlement sexuel est accepté comme une fatalité, comme la condition sine qua non pour qu'Isabel continue à travailler et à apporter de l'argent à la collectivité que forme ce groupe de travailleurs obligé, pour des raisons financières, de partager une même maison. Or, si ces employés apparaissent comme des victimes d'un système économique, ils deviennent dès le second chapitre ceux qui vont exploiter Gloria, l'une des colocataires. Cette dernière ayant démissionné du supermarché, devient la domestique de la maison. Elle n'est pas payée et subit à son tour un harcèlement de la part de ses «camarades » qui exploitent le statut dégradé qu'elle a au sein de la "communauté». La construction du roman met en relief le cercle vicieux qui s'est instauré: chaque chapitre dévoile la cruauté du personnage qui était auparavant victime de la cruauté des autres. C'est ainsi que Gloria dénonce au chapitre suivant Alberto auprès du superviseur car celui-ci voulait fonder un syndicat. Alberto est renvoyé du supermarché puis de la maison qu'il partageait avec ses camarades qui ne veulent pas que leur nom soit associé à celui d'un fauteur de trouble.

La caricature sociale est évidente. Les employés se révoltent non pas contre la précarité de leur statut mais contre celui qui s'y oppose. L'absence de lutte se manifeste par un refus ou une impossibilité de s'atteler aux véritables causes des problèmes. Ainsi, lorsque Gabriel ressent des douleurs répétées au bras, il ne mentionne pas la pénibilité de son travail mais accuse le bébé d'Isabel qui l'empêche de dormir la nuit. L'intériorisation par le 
groupe des normes aliénantes conduit à un enfermement psychologique dont les symptômes sont multiples. Pensons à Andrés qui reproduit dans la maison l'espace du supermarché, à Isabel qui, épuisée, reste prostrée ou à Gabriel qui se sent «fóbico a los clientes, a los paquetes, fóbico a sí mismo » (356). Le champ lexical de la pathologie envahit de nouveau le texte et traduit le profond malaise qui affecte chacun des personnages.

A la fin du roman, ne supportant plus la dureté de leurs conditions de travail et de vie, le groupe sombre dans la drogue. Tous sont licenciés par Enrique, devenu superviseur. L'expulsion de leur travail s'accompagne de l'expulsion de la maison conformément aux règles qu'ils avaient collectivement énoncées: n'accepter dans leur communauté ni chômeurs ni malades.

Chacun des deux chapitres s'achève donc sur une marginalisation croissante des narrateurs: le narrateur de la première partie s'attend à être licencié tandis que les narrateurs de la seconde partie vont grossir le rang des vagabonds urbains. La construction du roman qui met en œuvre un narrateur individuel puis un narrateur collectif, suggère bien entendu une portée plus générale des trajectoires des personnages mis en scène. Mano de obra reflète alors un positionnement éthique de la romancière mis en relief par le paratexte.

Le titre de la première partie «El despertar de los trabajadores (Iquique, 1911)» semble évoquer un sursaut de la classe ouvrière. De même, chaque sous-titre de la première partie renvoie à une publication anarchiste ou socialiste accompagnée de l'année de sa fondation. Il est à noter cependant que les dates de publication qui apparaissent ne sont pas classées chronologiquement et passent de 1918 à 1909 puis 1924 ce qui suggère peutêtre qu'il n'y a pas eu de montée en puissance constante des mouvements ouvriers. D'autre part, le décalage est patent entre les références à des mouvements ouvriers d'envergure dans le passé et le présent auquel renvoie la narration. Le premier sous-chapitre évoque une publication révolutionnaire par le biais du titre "Verba roja (Santiago, 1918)». Or, le début in medias res montre une frénésie d'achat ainsi que le harcèlement dont est victime le narrateur autodiégétique. Le procédé de décalage entre le titre et le contenu du chapitre souligne l'absence de revendications sociales dans une société individualiste et centrée sur la consommation. D'où la phrase de Sandra Cornejo mise en épigraphe et qui résonne tel un avertissement: "Algunas veces, por un instante, la historia debería sentir compasión y alertarnos» (251). 
Le second chapitre «Puro Chile (Santiago, 1970)» constitue une claire référence à l'hymne national chilien ainsi qu'à un journal qui a vu le jour en 1970, sous la présidence de Salvador Allende, avant d'être mis à sac en 1973, sur ordre d'Augusto Pinochet. Les sous-chapitres qui suivent font référence à des scènes de la vie quotidienne; par exemple: "Gloria va a dormir en la pieza de atrás» ou encore "Sonia lloró en el baño». Toute référence politique ouverte a disparu. La domination économique devient tacite parce que le groupe de narrateurs n'est pas en mesure d'élaborer une quelconque réflexion et parce que le terme décisif de "marché» est symboliquement effacé du texte: il est toujours question par exemple du «súper», jamais du «supermercado»...

Cette situation présente apparaît comme une conséquence et un prolongement du passé. Tout se passe comme si les tortures du système dictatorial avaient disparu au profit d'un système certes démocratique mais qui continue d'exercer une violence d'ordre économique sur certaines parties du corps social. Un lien de continuité s'établit entre El cuarto mundo (1988) et Mano de obra. Dans El cuarto mundo, l'enfermement croissant des personnages renvoyait à l'oppression du régime dictatorial. A la fin du roman, dans une ville où tout était à vendre, la sœur jumelle incestueuse donnait naissance à une petite fille-livre dont la narratrice affirmait, et c'était la dernière phrase de l'œuvre: «la niña sudaca irá a la venta» (Eltit 1988: 245). Mano de obra semble s'inscrire dans le prolongement chronologique de El cuarto mundo. La petite fille qui allait être vendue dans El cuarto mundo évoque le bébé d'Isabel dans Mano de obra, ce bébé symboliquement dépourvu de nom, symboliquement dépourvu de père et qui porte déjà les stigmates du corps social malade auquel il appartient. El cuarto mundo semblait annoncer l'avènement d'une société dominée par le marché, Mano de obra est centré sur sa toute-puissance. D'autre part, l'écrivaine affiche une volonté de s'éloigner d'écrits politiquement corrects: les employés marginalisés laissent se détacher une vision du Chili bien éloignée de celle du «jaguar d'Amérique Latine».

\section{Échec et projet littéraire de l'auteure}

Dans Mano de obra comme dans d'autres œuvres de Diamela Eltit, l'échec qui domine les pages contribue à communiquer un sentiment de malaise. En effet, Diamela Eltit explique qu'elle n'aspire pas à réconforter le lecteur par des histoires au dénouement heureux mais à lui faire partager des doutes et à l'entraîner, et ce, depuis ses premières œuvres, dans des zones de fractures 
porteuses de sens. L'échec occuperait donc une place de choix dans le projet littéraire de l'auteure. Un bref regard sur la généalogie de son œuvre permet de mettre en évidence différents aspects des liens entre écriture et échec.

Sous la dictature, Diamela Eltit avait développé une prose obscure qui devenait à la fois le symptôme des souffrances liées à l'oppression et une protection contre les censeurs: ceux-ci ne comprenant pas ses textes, les laissaient circuler librement. La prose problématique et obscure qui caractérise son œuvre et qui met bien souvent le lecteur en situation d'échec était à l'origine une façon de résister à la censure et d'affirmer une voix autre que celle du discours autoritaire. Ce procédé se trouve, par exemple, dans $E l$ cuarto mundo publié en 1988.

Dans cette œuvre, le langage torturé et opaque des personnages est une expression masquée de la souffrance liée à l'oppression. La perversion du discours symbolise l'impossibilité de s'exprimer clairement et de se justifier. Les gesticulations des protagonistes sont un moyen de montrer une douleur qui ne peut être verbalisée. La folie devient une manière de présenter des plaintes comme s'il s'agissait de divagations. Or, à la fin de l'œuvre, la sœur jumelle incestueuse qui donne naissance à une petite fille-livre destinée à la vente, est désignée par le nom de diamela eltit. De façon fort symbolique, l'auteur perd les majuscules de son nom, sombrant dans la désintégration qui a touché chacun des personnages: "Lejos, en una casa abandonada a la fraternidad, entre un 7 y un 8 de abril, diamela eltit, asistida por su hermano mellizo, da a luz una niña. La niña sudaca irá a la venta» (245).

L'enfant "sudaca» est le fruit d'une fraternité mise en danger par le pouvoir politique et l'ordre économique mondial... Cependant, cette dernière phrase marque la survie de la fraternité dans un contexte menaçant. Le terme péjoratif «sudaca» est récupéré dans la narration pour être assumé et devenir une revendication identitaire et un signe de non-acceptation de l'ordre mondial. La narratrice de la deuxième partie, évoquant ses désirs d'écriture avait affirmé: «Quiero escribir una obra sudaca, terrible y molesta" (Eltit 1988: XX). Or, sa narration et le roman tout entier peuvent être considérés comme des œuvres «sudacas», terribles et scandaleuses qui élaborent, à partir d'un échec social et politique, un discours transgressif.

De même, dans El Padre Mío, Diamela Eltit décentre la figure de l'auteure. En effet, ce livre, difficile à définir, est composé d'une présentation de Diamela Eltit puis de la transcription des discours d'un vagabond dans les rues de Santiago du Chili. Diamela Eltit livre de façon directe et violente le discours pathologique d'un individu marginalisé qui, pour elle, synthétise 
le Chili de son temps. La première partie de cet ouvrage correspond à des réflexions théoriques et des doutes de l'auteure tandis que la seconde partie suggère une filiation meurtrie.

A trois reprises Diamela Eltit a enregistré le discours délirant du SDF qu'elle avait rencontré. L'une des nombreuses questions qu'elle se posait était de savoir comment aborder cette réalité marginale alors qu'elle n'était ni anthropologue ni sociologue. Comment pouvait-elle faire apparaître le sens qu'elle trouvait au discours du SDF? Quatre années durant, Diamela Eltit a cherché quelle forme donner à ses réflexions et aux enregistrements qu'elle avait en sa possession. Ce n'est qu'en 1989 qu'est paru le petit livre $E l$ Padre Mío dans lequel Diamela Eltit met à nu sa démarche et les problèmes auxquels elle s'est trouvée confrontée. Elle y expose le sentiment d'échec qui l'anime en tant qu'écrivain face à une réalité sociale qu'elle a conscience de ne modifier en aucune façon. Rejetant les prismes théoriques prédéterminés, l'auteure a finalement opté pour une démarche littéraire reposant sur un vagabondage intellectuel. Ses réflexions sont donc livrées telles qu'elles, associant librement les termes pour faire jaillir un sens. A la page 16, la romancière cède la parole au SDF dont elle transcrit, sans le moindre commentaire, le discours délirant. Or, la démarche littéraire qu'elle a lancée incite le lecteur à lire le témoignage du SDF comme partie d'une œuvre d'art, se souciant plus du sens et de la forme de son discours que d'une quelconque exactitude historique.

Commence alors la deuxième partie del'œuvre, consacréeà la transcription de trois discours du vagabond. El Padre Mío est convaincu d'avoir joué un rôle décisif dans l'histoire récente du Chili et croit fermement qu'il va être amené à occuper de nouveau un rôle de premier plan. Il est atteint d'une forte paranoïa: il aurait été persécuté par le passé du fait de ce qu'il savait. On aurait essayé de le faire taire en l'enfermant dans un hôpital psychiatrique après avoir fait disparaître toute sa famille. D'après lui, «el Rey Jorge » détient le pouvoir et dispose du soutien de «El Padre mío » qui donne des ordres illégaux dans le pays. Un vaste complot se fait jour dans lequel l'identité des puissants se trouble. Il est constamment question de politique liée à l'économie et à la violence.

Le vagabond se trouve, par le biais de la construction qu'est l'ouvrage, intimement lié à l'auteure. L'ambiguïté du titre est évidente: la couverture du livre qui joint le nom de la romancière, une photo de l'homme et le titre El Padre Mío pourrait faire penser à un écrit autobiographique. De même, les premiers mots du texte «j'ai fait la connaissance de Mon Père en 1983» 
suggèrent une histoire familiale fracturée, marquée par des retrouvailles tardives. Mais, à la lecture de la présentation de l'auteure, il devient évident qu'aucun lien de parenté réel n'unit Diamela Eltit au SDF avec lequel elle s'entretient. C'est cependant elle qui, ignorant comment s'appelle le vagabond, le nomme El Padre Mío. Ces mots sont puisés dans le flot de paroles du clochard. «El Padre Mío» est en effet une expression qui revient constamment dans les accusations du SDF. Il désigne, pour ce dernier, un personnage haut-placé, doté d'un fort pouvoir de nuisance. Or, Diamela Eltit reprend ce nom pour désigner le SDF et en fait le titre de son écrit. Quel est le sens de cette filiation fictive qu'établit Diamela Eltit ? Par le biais de cette opération, l'auteure établit des liens de filiation entre elle et le vagabond: tous deux sont rebaptisés dans le cadre de cette création qui les unit. La notion de paternité et par conséquent de filiation, telle que Diamela Eltit la met en valeur ici, est intimement liée à un contexte politique et historique.

Les discours du SDF laissent en effet penser qu'il est une victime de la répression. Se présenter comme fille de cet homme équivaut à assumer une identité meurtrie et marginale construite en opposition au discours monolithique et oppressant du régime dictatorial. C'est également une façon de renier le père de la Patrie de l'époque. Or, le fait de prendre le nom du personnage tout-puissant dans le discours du clochard, de l'en déposséder pour le donner au SDF montre un parti pris de l'auteure, une réorganisation symbolique au sein de la construction proposée. Par ailleurs, Diamela Eltit se définit dans bien des entretiens comme la fille d'un milieu familial problématique, comme la fille de «son père et de ses pénuries.» Or, dans cet écrit, Diamela Eltit se présente de nouveau comme fille d'un père et de ses pénuries mais le père et la fille dépassent désormais l'histoire individuelle pour atteindre une dimension collective.

De fait, la filiation fictive que crée l'écrivain donne le jour à une fragile résistance liée à la rencontre et à la combinaison des deux voix du père et de la fille. Comme le remarque Michaël Lazzara, dans la conception patriarcale, c'est sur le père que repose l'ordre et le discours. Face au discours autoritaire dominant s'installe le discours de la fille qui porte le discours du père que nul n'écoute. De cette rencontre fille-père naît un ouvrage fruit de deux recherches: celle de la romancière en quête de sens et celle du SDF dans l'attente de quelqu'un disposé à recueillir son témoignage. De cette rencontre porteuse de sens est né ce petit livre qui s'oppose au silence de la dictature, ainsi qu'à l'idée d'ordre imposé par le régime. Du discours irrationnel du SDF naît, selon les termes de Diamela Eltit, une vérité 
malade à travers laquelle s'affirment des liens indissolubles entre politique, économie et violence. Dans le cadre fixé par l'écrivaine, les divagations du vagabond permettent d'approcher une forme de vérité tout en étant un reflet du traumatisme des victimes du régime et un discours opposé à l'histoire officielle du moment.

Cependant, sur le plan du cheminement littéraire de Diamela Eltit, l'idée de filiation peut acquérir une autre dimension. Si l'on regarde attentivement les dates des entretiens, antérieures à bien des travaux de la romancière, on peut penser que le discours schizophrène du Padre Mío a été une source d'inspiration pour des romans de Diamela Eltit, El cuarto mundo par exemple, publié en 1988 ou même Mano de obra. Dans ce sens, le vagabond aurait peut-être une part de paternité à revendiquer dans certaines œuvres de Diamela Eltit. Cette dernière bousculerait alors bien des conventions littéraires. Elle remettrait en cause son propre statut d'écrivain : peut-elle être considérée comme l'auteure à part entière d'un ouvrage dont elle n'a rédigé que quelques pages? D'autre part, sa source d'inspiration sur le plan de la construction des phrases et de la pathologie textuelle n'émanerait-elle pas d'un SDF qu'elle a été la seule à écouter et qui lui a dicté le titre de son écrit? C'est donc bien d'un double échec que naît cet ouvrage de Diamela Eltit: celui d'une auteure qui peine à trouver des modes d'expression et d'action qui la satisfassent, celui de la personne qui a inspiré son œuvre et qui se trouve dans une situation d'échec personnel, économique et politique.

Mano de obra s'inscrit dans cette démarche littéraire qui réfléchit sur les liens délicats qui unissent la créatrice à sa création. Diamela Eltit y manie un langage problématique. Dans la seconde partie, le groupe d'employés s'exprime de façon extrêmement vulgaire. La romancière joue alors, de façon provocatrice, sur les limites du langage pouvant être considéré comme littéraire. Les termes très grossiers sous la plume d'une auteure de renom peuvent sembler déplacés. Et de fait, cette dernière opère un déplacement et attire les spécialistes de littérature (que sont la plupart de ses lecteurs) vers les secteurs les plus marginaux, les incitant à chercher, de même que dans $E l$ Padre Mío, un sens à des discours aliénés et violents. L'auteure s'efface devant des discours de la rue; elle brise par là-même les stéréotypes en matière d'écriture féminine et s'oppose à l'ordre artificiel et aseptisé d'une société de supermarché. Cependant, la démarche et les doutes de la romancière apparaissent en filigrane dans le texte. La première partie se terminait par: «Veinticuatro (horas). Qué importa la inminencia del despido. Hay que 
poner fin a este capítulo» (Eltit 2002: 295). Ces mots trouvent un écho dans la dernière phrase du roman: «Demos vuelta la página » (360).

Les deux phrases sont ambiguës: elles peuvent se comprendre sur le plan diégétique comme un constat d'échec des narrateurs ou au contraire comme un élan de révolte visant à mettre un terme à une situation aliénante. Mais elles peuvent également être lues comme une référence métatextuelle, comme une allusion au livre dans sa matérialité et à l'écriture d'une œuvre qui s'impose à son auteure et s'achève sans avoir eu d'impact sur la réalité. De fait, le titre est polysémique. Mano de obra renvoie aux employés et se réfère également à l'acte de création, à la main qui écrit une œuvre. Cette polysémie réunit alors sous un même signe la main d'œuvre exploitée et la créatrice.

Pour conclure, Diamela Eltit qui remet en cause bien des schémas tant politiques que littéraires, compare ses livres à des châteaux de sable:

Tal como los niños construyen cosas, como escritora quiero jugar con los retazos y pedazos para hacer el castillo de arena que es mi libro, sin desconocer su carga social, su dramaticidad y su conexión, digamos, con unas historias siempre difíciles, y en algunos casos hostiles (Lazzara 2002: 124).

Cependant, ces textes que leur auteure définit comme des lieux de fractures du langage et du sens aspirent modestement à entraîner le lecteur hors des sentiers battus et à ouvrir de faibles brèches dans les discours dominants.

\section{Bibliographie}

Eltit, Diamela, 1989, El Padre Mío, Santiago de Chile, LOM Ediciones.

Eltit, Diamela, 2000, Emergencias, escritos sobre literatura, arte y política, Santiago de Chile, Planeta/Ariel.

Eltit, Diamela, 2004, "Mano de obra» in Tres novelas, México, Fondo de cultura económica.

Eltit, Diamela, 2004, «El cuarto mundo» in Tres novelas, México, Fondo de cultura económica.

Carreño Bolívar, Rubí, 2009, Diamela Eltit: redes locales, redes globales, Santiago de Chile, Nuevos hispanismos.

Lazzara, Michaël, 2002, Los años de silencio: conversaciones con narradores chilenos que escribieron bajo dictadura, Santiago de Chile, Editorial Cuarto Propio. 
Lazzara, Michaël, 2007, Prismas de la memoria: narración y trauma en la transición chilena, Santiago de Chile, Editorial Cuarto propio.

Lértora, Juan Carlos, 1993, Hacia una poética de literatura menor: la narrativa de Diamela Eltit, Santiago de Chile, Editorial Cuarto propio. 